\title{
Sirtuin 3 induces apoptosis and necroptosis by regulating mutant 553 expression in small-cell lung cancer
}

\author{
XINYU TANG ${ }^{1}$, YANG LI $^{1}$, LONG LIU ${ }^{2}$, RUI GUO ${ }^{1}$, PING ZHANG ${ }^{3}$, YIHE ZHANG ${ }^{4}$, \\ YONG ZHANG $^{1}$, JIA ZHAO ${ }^{1}$, JING SU${ }^{1}$, LIANKUN SUN ${ }^{1}$ and YANAN LIU ${ }^{1}$ \\ ${ }^{1}$ Department of Pathophysiology, Prostate Diseases Prevention and Treatment Research Center, \\ School of Basic Medical Sciences, Jilin University; ${ }^{2}$ Department of Cardiovascular Medicine, \\ China-Japan Union Hospital, Jilin University, Changchun, Jilin 130021; ${ }^{3}$ Department of Basic Medical Sciences, \\ Medical School, Xiangyang Vocational and Technical College, Xiangyang, Hubei 441050; ${ }^{4}$ Department of Neurology, \\ The First Hospital of Jilin University, Jilin University, Changchun, Jilin 130021, P.R. China
}

Received June 26, 2018; Accepted November 1, 2019

DOI: $10.3892 /$ or.2019.7439

\begin{abstract}
Mutation of the p53 tumor suppressor frequently occurs in lung cancer, and can be as high as $75-90 \%$ in small-cell lung cancer. Mutant p53 (mtp53) can inhibit the wild-type p53 protein, disrupting its tumor suppressor functions. In addition, mutant p53 often acquires the functions of an oncogene. Post-translational modification of the p53 protein is important for its transcriptional and tumor suppressive functions. We previously revealed that high levels of mutant p53 expression were associated with reduced expression of the deacetylation enzyme sirtuin 3 (SIRT3) in lung cancer tissues. Given this negative correlation between p53 and SIRT3 expression, and given that SIRT3 is a deacetylase, we speculated that SIRT3 participates in the post-translational modification of mutant $\mathrm{p} 53$, regulating its stability and function, thereby inhibiting the growth of lung cancer cells. Light microscopy, MTT and flow cytometric assays revealed that SIRT3 overexpression inhibited growth and promoted apoptosis in NCI-H446 human small cell lung cancer cells. SIRT3 overexpression also resulted in necroptosis, and this could be partially reversed following cell treatment with the necroptosis inhibitor necrostatin-1 (Nec-1), which could restore certain cells to survive. Western blotting assays revealed that SIRT3 overexpression resulted in the reduced expression and half-life of mutant p53, indicating that SIRT3 decreases mutant p53 stability. Proteasome inhibitor experiments revealed that the decrease in mutant p53 stability was a result of increased proteasomal degradation of the protein. Immunoprecipitation studies revealed that ubiquitination of mutant p53 was elevated in SIRT3-overexpressing cells, indicating that SIRT3 affected
\end{abstract}

Correspondence to: Professor Yanan Liu, Department of Pathophysiology, Prostate Diseases Prevention and Treatment Research Center, School of Basic Medical Sciences, Jilin University, 126 Xinmin Street, Changchun, Jilin 130021, P.R. China E-mail:ynliu@jlu.edu.cn

Key words: SCLC, mutant p53, SIRT3, apoptosis, necroptosis ubiquitination-mediated protein degradation. In the present study, it was therefore revealed that SIRT3 can inhibit the growth of human small-cell lung cancer cells by promoting apoptosis and necroptosis. It was also revealed that SIRT3 expression could regulate the stability of mutant p53 by controlling ubiquitination-mediated proteasomal degradation of the protein. SIRT3 expression may therefore play an important role in the growth of mutant p53-associated lung cancer.

\section{Introduction}

Lung cancer is a highly malignant disease, and lung cancer-associated deaths and morbidity are increasing yearly. The World Health Organization divides lung cancer into small-cell lung cancer (SCLC) and non-small cell lung cancer (NSCLC), with small-cell lung cancer accounting for 13 percent of lung cancer patients (1). Small-cell lung cancer is characterized by rapid growth and high invasiveness. If the patient is not treated in a timely and effective manner, they will likely die within a few months. For decades, clinical trials for a variety of treatment regimens have failed to find a cure for small-cell lung cancer, and most patients relapse or succumb to metastasis after treatment $(2,3)$. Effective therapeutic targets and solutions remain an unmet need in the treatment of small-cell lung cancer, largely because we still know little concerning the molecular mechanisms driving the development of this disease.

The TP53 tumor suppressor gene is mutated in the majority of human tumors, and mutant p53 protein is highly expressed in $>50 \%$ of these tumors, inhibiting the activity of wild-type p53 and acquiring oncogenic functions. The incidence of TP53 mutation in small-cell lung cancer is as high as $75-90 \%$ (4). Mutation inhibits the normal transcriptional activity of p53, thereby preventing the ability of p53 to protect cells from malignant transformation (5). Regulation of p53 protein translation is considered to be the main mechanism by which the transcriptional activity and tumor suppressive functions of the protein are regulated. Sirtuin 3 (SIRT3) is a member of the Sirtuin family of nicotinamide adenine dinucleotide $\left(\mathrm{NAD}^{+}\right)$-dependent deacetylases (6). SIRT3 is a major 
mitochondrial stress-reactive protein, and participates in the binding and deacetylation of enzymes that regulate important mitochondrial functions. In recent years, an increasing number of studies have revealed that SIRT3 plays an important role in tumor biology (7). The function of SIRT3 may vary depending on cell and tumor type, and SIRT3 may therefore exhibit the characteristics of either an oncogene or tumor suppressor gene. In recent years, the association between SIRT3 and p53 signaling has received increasing attention. There are several studies that have reported the regulation of 553 post-translational modification by SIRT3. Zhang et al proposed that overexpression of SIRT3 upregulated the protein level of p53 by attenuating MDM2-mediated p53 degradation in liver cancer (8). The balance between acetylated and deacetylated p53 within the cell determines the overall transcriptional activity of the protein, and this has consequences for the downstream transcription of p53-responsive genes associated with cancer. Therefore, it is important to explore the role of SIRT3-dependent deacetylation in the regulation of p53. Indeed, some studies suggest that acetylation also affects the activity of mutant p53 (9-11).

In a previous study using the human non-small cell lung cancer cell line A549, we revealed that SIRT3 overexpression could both enhance the expression and reduce the acetylation of p53 within the cell, indicating that SIRT3 may affect the balance of acetylated-to-deacetylated p53. In addition, our clinical data revealed a negative correlation between mutant p53 and SIRT3 expression (unpublished data). We therefore speculate that SIRT3 may be involved in the post-translational modification of mutant p53, thereby affecting the stability of the protein and, consequently, the growth of lung cancer cells.

In the present study, human small-cell lung cancer cell line NCI-H446, which is positive for mutant p53, was used to examine the effects of SIRT3 expression on cell behavior and mutant $\mathrm{p} 53$ expression. These findings provide additional insights into the regulation of mutant p53 in small-cell lung cancer, highlighting new possibilities for the development of treatment strategies for this disease.

\section{Materials and methods}

Cell culture. NCI-H446 cells were obtained from ATCC and stored under liquid nitrogen at the Prostate Diseases Prevention and Treatment Research Center, Jilin University, Changchun, China. The cells were cultured in Dulbecco's modified Eagle's medium (DMEM; Gibco; Thermo Fisher Scientific, Inc.) supplemented with $10 \%$ fetal bovine serum (Biological Industries). Cells were grown in a humidified incubator maintained at $37^{\circ} \mathrm{C}$ and supplied with $5 \% \mathrm{CO}_{2}$. Cells were passaged by trypsinization (0.25\% EDTA-free trypsin; Sangon Biotech Co., Ltd.) and reseeded in new culture dishes at one half of their original density. The day after cultures had reached a confluency of 70\%, cells were transiently transfected with NC or SIRT3 expression constructs. RNA and protein were then extracted from cells $48 \mathrm{~h}$ post-transfection, for use in downstream experiments.

MTT assay. NCI-H446 cells were seeded in 96-well plates at a density of $6 \times 10^{3}$ cells/well. After $24 \mathrm{~h}$, the cells were transfected with either empty control plasmid (NC group) or plasmid encoding SIRT3 (SIRT3 group). Plasmids were constructed by Shanghai GeneChem Co., Ltd. Cells were then maintained under normal culture conditions until analysis of viability by MTT assay. To assess viability, $20 \mu \mathrm{l}$ of 3-(4,5-dimethylthiazol-2-yl)-2,5-diphenyltetrazolium bromide (MTT; Sangon Biotech Co., Ltd.) was added per well of the 96-well plate. After a 4-hour incubation, $150 \mu \mathrm{l}$ dimethyl sulfoxide (Sangon Biotech Co., Ltd.) was then added to each well. The absorbance of each well at $490 \mathrm{~nm}$ was then measured using a FLUOstar Omega microplate reader (BMG LABTECH). Cell viability was assessed at 24,48 and $72 \mathrm{~h}$ post-transfection.

Western blotting. To prepare protein lysates for western blotting assays, cultured cells were washed with PBS, detached with a cell scraper and then lysed in 100-200 $\mu$ l RIPA buffer (Beyotime Institute of Biotechnology) containing the protease inhibitor phenylmethylsulfonyl fluoride (Roche Diagnostics). Cell lysates were then sonicated on ice to disrupt protein aggregates by using ultrasonic disruptor UD-201 (TOMY SEIKO Co., Ltd.). Samples were then clarified by centrifugation at $15,000 \mathrm{x}$ for $20 \mathrm{~min}$ at $4^{\circ} \mathrm{C}$, and the supernatants were retained. Clarified protein lysates ( $20 \mu \mathrm{g}$ per lane) were then resolved by electrophoresis on $12 \%$ polyacrylamide gels and subsequently transferred onto polyvinylidene fluoride membranes (EMD Millipore). The membranes were blocked in Tris-buffered saline $/ 0.5 \%$ Tween- 20 (TBST) containing 5\% non-fat milk for $1 \mathrm{~h}$ at room temperature, washed three times in TBST and then incubated with primary antibodies overnight at $4^{\circ} \mathrm{C}$. The membranes were then washed three times in TBST before incubation with secondary antibodies for $1 \mathrm{~h}$. After washing three times with TBST, the membranes were incubated with Hypersensitive ECL chemiluminescence reagent (Beyotime Institute of Biotechnology), and immunoreactivity was subsequently detected using a Syngene HR chemiluminescence imaging system (Synoptics). Primary antibodies, their respective dilutions and manufacturers, were as follows: anti-SirT3 (1:1,000; clone D22A3; product \#5490), cleaved caspase-3 (1:1,000; product \#9664), cleaved caspase-9 (1:1,000; product \#9509), and BID (1:1,000; product \#2002S) were purchased from Cell Signaling Technology, Inc.; anti- $\beta$-actin $(1: 1,000$, cat. no. 66009-1-Ig) was obtained from Proteintech Group; anti-Bcl-2 (1:1,000, ab32124), anti-RIP (1:1,000; product code ab125072), RIP3 (1:1,000; product code ab152130), MLKL (1:1,000; product code ab183770), p-MLKL (1:1,000; product code ab196436), LC3 II (1:1,000; product code ab48394), and mutant p53 antibodies [Y5] (1:1,000; product code ab32049) were obtained from Abcam; anti-Mcl-1 (1:200; cat. no. sc-819), Bax (1:200; cat. no. sc-7480), and Ubiquitin (1:200; sc-8017) antibodies were purchased from Santa Cruz Biotechnology, Inc.; anti-Beclin-1 (1:1,000; cat. no. 612112) was obtained from BD Biosciences. Horseradish peroxidase goat anti-mouse (1:2,000; cat. no. SA00001-1) and goat anti-rabbit (1:2,000; cat. no. SA00001-2) antibodies were purchased from Proteintech Group.

Caspase activity. Caspase-Glo 3/7 Assay (Promega Corporation) were used to determine cellular caspase-3/7 activities according to the manufacturer's instructions.

Reverse transcription PCR (RT-PCR). Total RNA was extracted from tissue samples and cell lines using TRIzol 
Table I. Primer sequences for reverse transcription polymerase chain reaction.

\begin{tabular}{lll}
\hline Gene & \multicolumn{1}{c}{ Forward primer sequence } & \multicolumn{1}{c}{ Reverse primer sequence } \\
\hline GAPDH & 5'-AGAAGGCTGGGGCTCATTTG-3' & 5'-AGGGGCCATCCACAGTCTTC-3' \\
$T P 53$ & 5'-CCATCTACAAGCAGTCACAG-3' & 5'-CAAATCTACAAGCAGTCACAG-3' \\
$B C L 2$ & 5'-GACTTCGCCGAGATGTCCAGC-3' & 5'- GCGTTACGATCGCCTCCATCA-3' \\
BAX & 5'-CGGCGAATTGGAGATGA ACTG-3' & 5'-AGCAAAGTAGAAGAGGGCAACC-3' \\
CASP3 & 5'-ATGGACAACAACGAAACCTCCGTG-3' & 5'-CCACTCCCAGTCATTCCTTTTAGTG-3'
\end{tabular}

Table II. Reaction conditions for reverse transcription polymerase chain reaction.

\begin{tabular}{lllll}
\hline Gene & Denaturation & Annealing & Extension & No. of cycles \\
\hline GAPDH & $94^{\circ} \mathrm{C}, 30 \mathrm{sec}$ & $55^{\circ} \mathrm{C}, 30 \mathrm{sec}$ & $55^{\circ} \mathrm{C}, 30 \mathrm{sec}$ & 26 \\
SIRT3 & $94^{\circ} \mathrm{C}, 30 \mathrm{sec}$ & $55^{\circ} \mathrm{C}, 30 \mathrm{sec}$ & $55^{\circ} \mathrm{C}, 30 \mathrm{sec}$ & $72^{\circ} \mathrm{C}, 30 \mathrm{sec}$ \\
$T P 53$ & $94^{\circ} \mathrm{C}, 30 \mathrm{sec}$ & $56^{\circ} \mathrm{C}, 30 \mathrm{sec}$ & $72^{\circ} \mathrm{C}, 30 \mathrm{sec}$ & 30 \\
$B C L 2$ & $94^{\circ} \mathrm{C}, 30 \mathrm{sec}$ & $55^{\circ} \mathrm{C}, 30 \mathrm{sec}$ & $72^{\circ} \mathrm{C}, 30 \mathrm{sec}$ & 30 \\
$B A X$ & $94^{\circ} \mathrm{C}, 30 \mathrm{sec}$ & $54^{\circ} \mathrm{C}, 30 \mathrm{sec}$ & $72^{\circ} \mathrm{C}, 30 \mathrm{sec}$ & \\
CASP3 & $94^{\circ} \mathrm{C}, 30 \mathrm{sec}$ & $55^{\circ} \mathrm{C}, 30 \mathrm{sec}$ & &
\end{tabular}

(Invitrogen; Thermo Fisher Scientific, Inc.) based on standard protocols. NCI-H446 cells with SIRT3 overexpression treatment were cultured in a 6-well plate, washed twice with cold PBS and the culture medium was discarded. Then $500 \mu \mathrm{l}$ of TRIzol per well was added and incubation followed for $5 \mathrm{~min}$ at room temperature. Chloroform, isopropanol and $75 \%$ ethanol were added successively, and mRNA was obtained by drying at room temperature after $7,500 \mathrm{x} g$ centrifugation repeatedly. Subsequent generation of cDNA was performed using the EasyScript First-Strand cDNA Synthesis SuperMix kit according to the manufacturer's instructions (Beyotime Institute of Biotechnology). Briefly, in all reactions, 50-500 ng RNA was used as the starting template. The RT-PCR reaction was performed at $42^{\circ} \mathrm{C}$ for $30 \mathrm{~min}$ followed by an enzyme inactivation step at $85^{\circ} \mathrm{C}$ for $5 \mathrm{sec}$. Primer information and reaction conditions are presented in Tables I and II.

Flow cytometric analysis of apoptosis. NCI-H446 cells were seeded in 6 -well plates at a density of $3 \times 10^{5}$ cells/well. After $24 \mathrm{~h}$, the cells were transfected with either empty plasmid (NC group) or plasmid encoding SIRT3 (SIRT3 group). After $48 \mathrm{~h}$, the cells were collected, washed twice with PBS, and apoptosis was then evaluated using the FITC Annexin V Apoptosis Detection kit (BD Biosciences). Briefly, cells were resuspended in $100 \mu \mathrm{l}$ of $1 \mathrm{X}$ Binding Buffer before the addition of $5 \mu \mathrm{l}$ Annexin V-FITC and $5 \mu \mathrm{l}$ propidium iodide. The cells were then incubated in the dark for $15 \mathrm{~min}$. An additional $400 \mu \mathrm{l}$ of 1X Binding Buffer was added before analysis of apoptosis using a BD FACSCalibur flow cytometer (BD Biosciences).

Detection of mitochondrial membrane potential. Mitochondrial membrane potential was assessed using the Mitochondrial membrane potential assay kit with JC-1 (Beyotime Institute of Biotechnology), which accumulates in healthy mitochondria. NCI-H446 cells were seeded into 6-well plates at a density of $3 \times 10^{5}$ cells/well. After experimental treatments, cells were harvested by digestion with EDTA-free trypsin. The cells were then resuspended in $1 \mathrm{ml}$ serum-free DMEM containing $1 \mu \mathrm{l}$ JC-1 $(5 \mu \mathrm{g} / \mathrm{ml})$ and incubated at $37^{\circ} \mathrm{C}$ for $20 \mathrm{~min}$. At $3-5 \mathrm{~min}$ intervals, the cells were removed from the incubator and mixed by inversion to ensure uniform uptake of the dye. The cells were then pelleted by centrifugation at 1,500-2,000 x g for $5 \mathrm{~min}$, washed with PBS and then resuspended in $400 \mu \mathrm{l}$ DMEM. The fluorescence intensity of dye-labelled cells was analyzed using a BD FACSCalibur flow cytometer.

Proteasome inhibition assay. NCI-H446 cells were divided into five groups as follows; untransfected cells (CON group), cells transfected with empty plasmid (NC group), cells transfected with SIRT3 plasmid (SIRT3 group), cells treated with MG132 only (MG132 group), and cells transfected with SIRT3 plasmid and subsequently treated with MG132 (MG132+SIRT3 group). NCI-H446 cells were seeded into 6-well plates and $24 \mathrm{~h}$ later the appropriate groups were transfected with their respective plasmids and treated with MG132 inhibitor as indicated. After an additional $24 \mathrm{~h}$, the cells were collected and lysates were prepared for analysis of protein expression by western blotting.

Analysis of protein half-life. Cycloheximide (CHX) was used to evaluate changes in mutant p53 protein half-life. NCI-H446 cells were transiently transfected with a plasmid encoding SIRT3 $48 \mathrm{~h}$ prior to CHX experiments. Cells were then treated with $50 \mu \mathrm{g} / \mathrm{ml} \mathrm{CHX}$, and cell lysates were subsequently obtained at $0,0.5,1$, and 2-h time-points for analysis of protein expression by western blotting.

Immunoprecipitation assays. After NCI-H446 cells had been subjected to the indicated treatments, they were washed twice with PBS and lysed in RIPA buffer. Cell lysates were incubated for $30 \mathrm{~min}$ on ice with rotation. Lysates were then clarified by 
centrifugation at $14,000 \mathrm{xg}$ for $15 \mathrm{~min}$ at $4^{\circ} \mathrm{C}$, and the supernatants were subsequently retained and transferred to a fresh tube. Approximately 30-50 $\mu \mathrm{l}$ of clarified lysate was retained for analysis of input. Samples containing $1 \mathrm{mg}$ total protein were incubated with $2 \mu \mathrm{g}$ antibody overnight on ice. The following day, protein $\mathrm{A} / \mathrm{G}$-conjugated beads were added and the samples were then incubated for an additional night on ice. On the third day, the beads were pelleted by centrifugation for $5 \mathrm{~min}$ at $1,000 \mathrm{x} \mathrm{g}$ at $4^{\circ} \mathrm{C}$ and the supernatants were discarded. The beads were then washed 3-5 times with PBS. Finally, $60 \mu 1$ RIPA buffer was added to $20 \mu 1$ of bead slurry and $20 \mu \mathrm{l}$ of sample was used/well for subsequent polyacrylamide gel electrophoresis.

Animal experiments. Salmonella typhimurium A1-R is auxotrophic for arg and leu, which enables improved targeting and toxicity towards tumors, and this strain has previously been used in cancer treatment (12). Mouse-attenuated salmonella typhimurium $\mathrm{phoP} / \mathrm{phoQ}$ was used in this study to deliver expression constructs to tumor cells in vivo. Attenuated salmonella typhimurium was induced into competence. Competent cells $(100 \mu 1)$ were mixed with $1 \mu \mathrm{l}$ SIRT3 plasmid, and then transferred to a cold MicroPulser electroporation cuvette $(0.1-\mathrm{cm}$ gap). The cuvette was placed in a Gene Pulser Transfection Apparatus (product no. 1652100; Bio-Rad Laboratories, Inc.), and pulsed once. After hearing the beep, $1 \mathrm{ml} \mathrm{LB}$ liquid medium was immediately added into the cuvette. After gently resuspending the cells, they were transferred to a new centrifuge tube, cultured at $37^{\circ} \mathrm{C}$ for $1 \mathrm{~h}$, and shaked at 220 RPM. Then the cells were plated on LB plates with ampicillin at $37^{\circ} \mathrm{C}$ for $12 \mathrm{~h}$. The following parameters were used for electroporation: $2.5 \mathrm{kV}, 25 \mu \mathrm{F}, 200 \Omega, 5 \mathrm{msec}$.

The animal study was approved by the Animal Ethics Review Committee of Basic Medical Sciences, Jilin University, in accordance with the regulations of the Institutional Committee for the Care and Use of Laboratory Animals of the Experimental Animal Center of Jilin University.

A total of 12 male 4 to 6 -week-old BALB/C nu/nu nude mice weighing between 18-20 g were used for in vivo experiments. Animals were purchased from Huafukang Bioscience Co., Inc. Each mouse was injected subcutaneously with $2 \times 10^{6}$ cells in a final suspension volume of $100 \mu \mathrm{l}$. Tumor size was then monitored daily by Vernier caliper until they had reached approximately $5 \mathrm{~mm}$ in diameter. Ten tumor-bearing mice were then randomly divided into two groups; the PQ group (animals injected with attenuated Salmonella transformed with empty plasmid) and the PQ-SIRT3 group (animals injected with attenuated Salmonella transformed with SIRT3 plasmid). Mice were intraperitoneally injected every 14 days with $5 \times 10^{7} \mathrm{cfu}$ of PQ or PQ-SIRT3-harboring bacteria. After treatment, the behavior and changes in water intake and weight of the animals were monitored. The maximum length (L) and short diameter (W) of the tumor was measured by Vernier caliper to calculate the tumor volume using the formula $\mathrm{V}=0.52 \times \mathrm{L} \mathrm{x} \mathrm{W}^{2}$. The tumor growth index was used to record and plot tumor growth and was calculated using the formula: Tumor growth index $=\left(\mathrm{V}_{\mathrm{n}}-\mathrm{V}_{1}\right) / \mathrm{V}_{1}$ (where $\mathrm{V}_{1}$ is the tumor volume of the first day, and $V_{n}$ is the tumor volume of the nth day).

After 25 days of treatment, the nude mice were euthanized by cervical dislocation after intraperitoneal injection of $20 \%$ urethane $(0.1 \mathrm{ml}$ per mouse) and the tumors were removed, weighed and photographed. A small portion of each tumor was fixed in $10 \%$ formaldehyde solution for subsequent hematoxylin and eosin (H\&E) staining. The remaining tumors were stored in liquid nitrogen for subsequent RNA and protein extraction.

Histology and immunohistochemistry. The tumors were fixed in $10 \%$ formaldehyde solution for $48 \mathrm{~h}$, and then embedded in paraffin. Histological sections were prepared by standard conventional processing and stained with $\mathrm{H} \& \mathrm{E}$. The sections $(0.2 \mathrm{~mm})$ were incubated with $2 \%$ bovine serum albumin (BSA) for $30 \mathrm{~min}$ at RT to block nonspecifc binding, and then incubated with rabbit polyclonal anti-SIRT3 (1:200; product no. 2627; Cell Signaling Technology, Inc.) and anti-mutant p53 antibodies [Y5] (1:1,000; product no. ab32049), followed by incubation with goat anti-rat immunoglobulin $\mathrm{G}$ ( IgG; 1:400; cat. no. SA00001-15; ProteinTech Group, Inc.) for $1 \mathrm{~h}$ at RT. Between each incubation step, the sections were washed 3 times with Tris-buffered saline with Tween-20 (12.5 mM Tris/HCl, pH 7.6, $137 \mathrm{mM} \mathrm{NaCl}$ and 0.1\% Tween-20).

Statistical analysis. All the data were obtained after at least three independent experiments. Data are presented as the mean \pm standard deviation. A Student's t-test was used to compare two groups and ANOVA with Tukey post hoc test was used to enable multiple comparisons between groups. $\mathrm{P}<0.05$ was considered to indicate a statistically significant difference in data.

\section{Results}

SIRT3 overexpression inhibits the growth of NCI-H446 small-cell lung cancer cells. To study the effect of SIRT3 expression on the growth of small-cell lung cancer cells, NCI-H446 cells were transfected with either a control plasmid (NC group) or a plasmid encoding SIRT3 (SIRT3 group). Western blotting and RT-PCR analysis confirmed an increase in expression of SIRT3 in cells of the SIRT3 group, when compared with those of the $\mathrm{NC}$ group $(\mathrm{P}<0.01)$, indicating that the transfection was successful. A microscopy-based analysis of cell morphology revealed an increase in cell death among cells of the SIRT3-group at $24 \mathrm{~h}$ post-transfection (Fig. 1B). By $48 \mathrm{~h}$ post-transfection the difference in cell death between the control and SIRT3 groups was even more pronounced. SIRT3 overexpression promoted cell rounding and detachment, resulting in increased numbers of cells floating in the culture media. MTT assays revealed that cell viability in the SIRT3 group was reduced to $75 \%$ compared to the control group $24 \mathrm{~h}$ after transfection (Fig. 1C). By 48 and $72 \mathrm{~h}$ post-transfection, viability of the SIRT3 group was reduced to 43 and $20 \%$, respectively, indicating that overexpression of SIRT3 in lung cancer NCI-H446 cells inhibited the growth of NCI-H446 cells in lung cancer.

SIRT3 overexpression results in apoptosis of small-cell lung cancer NCI-H446. Cell apoptosis was detected by flow cytometry BD Accuri C6. The upper right quadrant represents late apoptosis and necroptosis, and the lower right quadrant represents early apoptosis. It can be observed from Fig. 2A that 

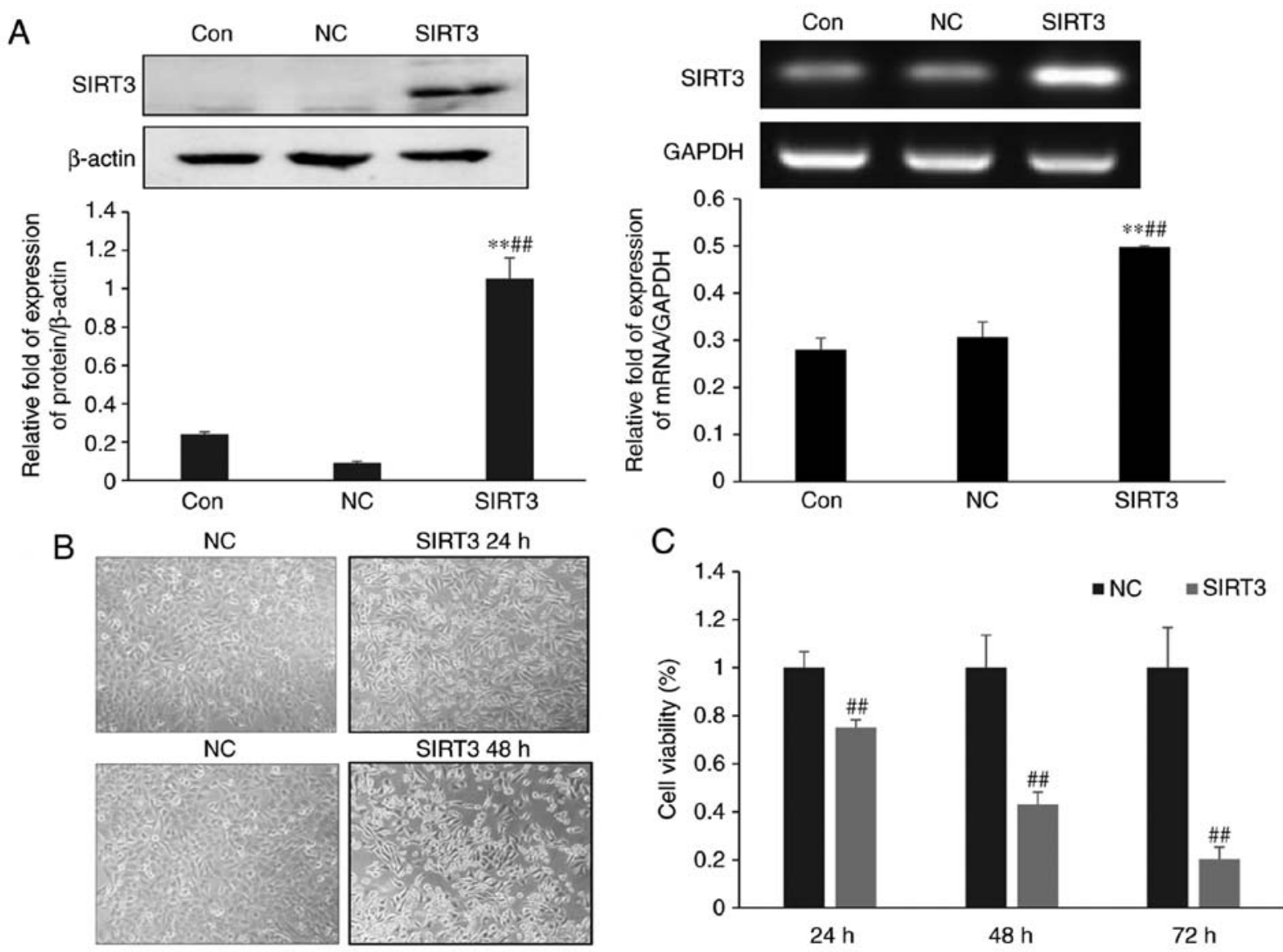

Figure 1. SIRT3 overexpression inhibits the growth of NCI-H446 small-cell lung cancer cells. (A) Confirmation of exogenous SIRT3 expression by RT-PCR and western blotting. (B) Representative light microscopic images demonstrating the effects of SIRT3 overexpression of cell morphology (magnification, x200). (C) NCI-H446 cell viability as determined by MTT assay. ${ }^{* *} \mathrm{P}<0.01$ vs. the control group; ${ }^{* \#} \mathrm{P}<0.01$ vs. the NC group. SIRT3, Sirtuin 3.

early apoptosis of the SIRT3 group was increased compared to the NC group, and late apoptosis and necroptosis were also increased $(\mathrm{P}<0.01)$. The mitochondrial membrane potential was also examined by flow cytometry, and it was revealed to be significantly decreased in the SIRT3-overexpressing cells when compared with the control (Fig. 2B; $\mathrm{P}<0.05$ ). The results of western blotting experiments revealed that the expression of the apoptosis-related proteins cleaved caspase-9, Bax, Bid, and cleaved caspase- 3 was significantly increased when compared with controls (Fig. $2 \mathrm{C} ; \mathrm{P}<0.01$ ), and that the expression of the anti-apoptotic proteins Bcl-2 and MCL1 was significantly decreased (Fig. 2C; P<0.01). RT-PCR analysis also revealed a significant increase in the expression of the pro-apoptotic genes TP53, BAX and CASP3 $(\mathrm{P}<0.05)$ and a decrease in the expression of the pro-survival gene $B C L 2(\mathrm{P}<0.01)$ in SIRT3-overexpressing cells (Fig. 2D). Furthermore, after $24 \mathrm{~h}$ of treatment with SIRT3 overexpression, a significant increase in caspase-3/7 activity was revealed in NCI-H446 cells (Fig. 2E).

SIRT3 overexpression promotes necrotic apoptosis in NCI-H446 small-cell lung cancer cells. Nec-1, an inhibitor of necrotic apoptosis, was used to determine whether SIRT3-dependent cell death involved the necrotic pathway. Twenty-four hours post-transfection with control or SIRT3 expression plasmids, NCI-H446 cells were treated with different concentrations of $\mathrm{Nec}-1$, and cell survival was subsequently assessed by MTT assay. This experiment revealed that Nec-1 treatment could partially reverse the effects of SIRT3-overexpression on cell death (Fig. 3A; $\mathrm{P}<0.01)$. However, the effects of Nec-1 treatment did not increase with increasing drug concentration. Western blot analysis of the expression of RIP1, RIP3, MLKL and p-MLKL, four important regulators of programmed necroptosis, revealed that their expression was significantly increased in SIRT3-overexpressing cells, when compared with controls (Fig. 3B; P<0.01). Collectively, these data indicated that SIRT3 overexpression promoted necroptosis in NCI-H446 cells.

SIRT3-dependent changes in mutant p53 expression and the underlying mechanism. Next, mutant p53 expression in SIRT3-overexpressing NCI-H446 cells was evaluated by western blotting. As revealed in Fig. 4A, mutant p53 protein levels were significantly decreased in cells overexpressing SIRT3, when compared with controls $(\mathrm{P}<0.05)$. SIRT3 overexpression resulted in a reduction in the half-life of mutant p53 protein (Fig. 4B). Therefore, the mechanism underlying this SIRT3-dependent decrease in mutant p53 expression was investigated. Protein degradation in eukaryotic cells is primarily controlled by the lysosomal and ubiquitin proteasome pathways. To determine whether the lysosomal pathway was implicated in mutant $\mathrm{p} 53$ degradation, the expression of the autophagy-associated proteins LC3 II and Beclin-1 were examined in SIRT3-overexpressing cells and it was revealed 

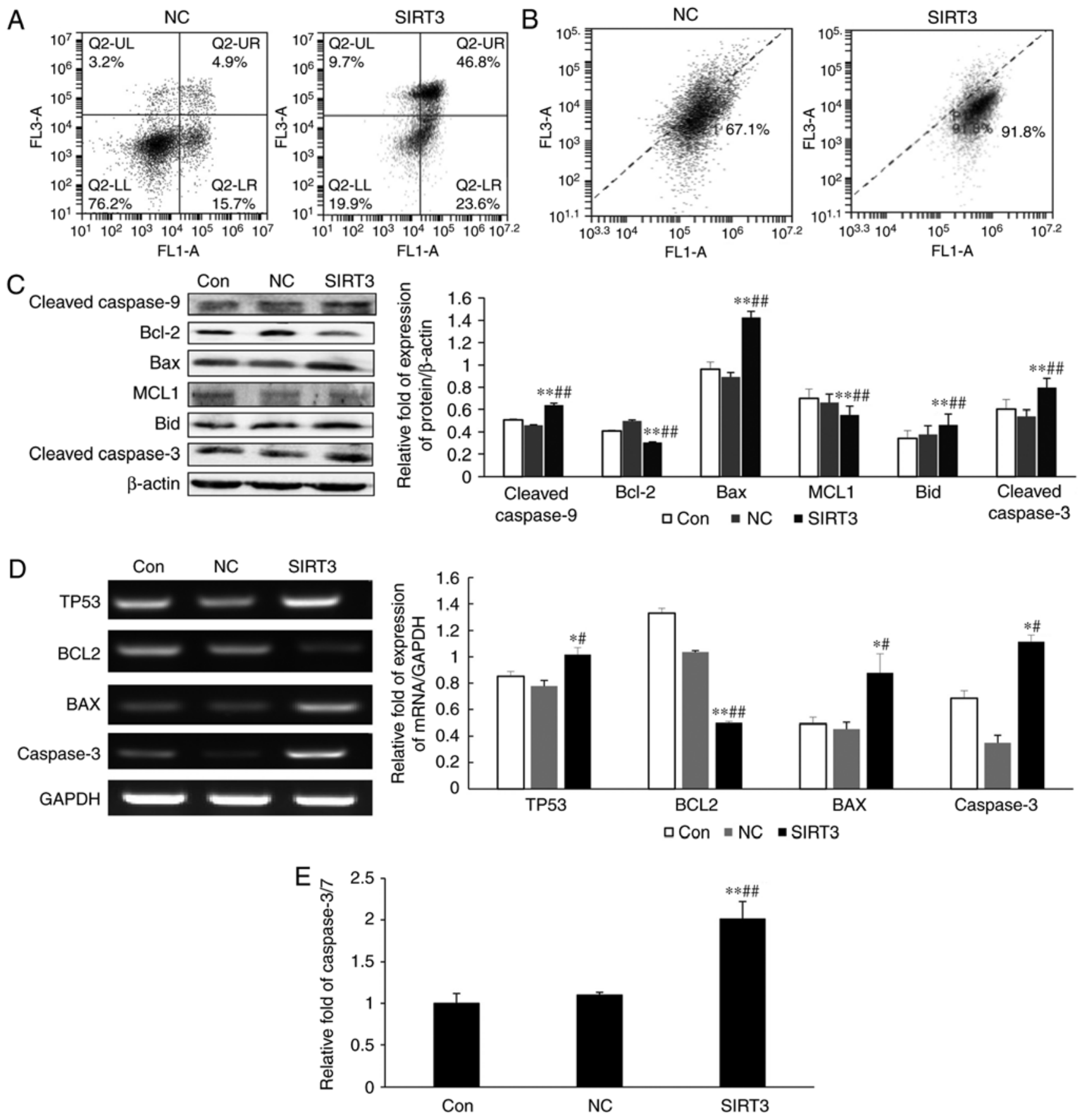

Figure 2. Effect of SIRT3 overexpression on NCI-H446 cell apoptosis. (A) Evaluation of apoptosis in control and SIRT3-overexpressing cell populations by FITC-Annexin V/PI staining-based flow cytometry. Increased apoptosis was observed following SIRT3-overexpression. (B) Flow cytometric analysis of the effects of SIRT3 overexpression on mitochondrial membrane potential. Mitochondrial membrane potential was decreased in SIRT3-overexpressing NCI-H446 cells $48 \mathrm{~h}$ post-transfection. (C) Western blot analysis of cleaved caspase-9, Bcl-2, Bax, MCL1, Bid, and cleaved caspase-3 protein levels in control and SIRT3-overexpressing cells. (D) RT-PCR was used to evaluate changes in TP53, BCL2, BAX and CASP3 gene expression between control and SIRT3-overexpressing cells. (E) Caspase-3/7 activities in NCI-H446 cells with SIRT3 overexpression. ${ }^{*} \mathrm{P}<0.05$ vs. the Con group; ${ }^{* *} \mathrm{P}<0.01$ vs. the Con group; ${ }^{\#} \mathrm{P}<0.05$ vs. the NC group; ${ }^{\#} \mathrm{P}<0.01$ vs. the NC group. SIRT3, Sirtuin 3.

that their levels remained unchanged, when compared with the control NC group (Fig. 4C). Therefore, it was hypothesized that mutant $\mathrm{p} 53$ was degraded by the ubiquitin proteasome pathway.

MG132 is a potent inhibitor of protein degradation mediated by the ubiquitin proteasome pathway (13). SIRT3-overexpressing NCI-H446 cells were treated with MG132 for $24 \mathrm{~h}$, and then protein lysates were prepared for analysis of mutant p53 expression. Western blot analysis revealed that mutant $\mathrm{p} 53$ protein expression in the $\mathrm{NC}+\mathrm{MG} 132$ group was significantly increased, when compared with the NC group (Fig. 4D; $\mathrm{P}<0.05$ ). An interaction between mutant p53 and SIRT3 was demonstrated by immunoprecipitation assay (Fig. 4E). Immunoprecipitation assays also revealed that mutant p53 in SIRT3-overexpressing cells was more highly ubiquitinated than in control cells. These data indicated that overexpression of SIRT3 promoted degradation of mutant $\mathrm{p} 53$ by the ubiquitin proteasome pathway. 


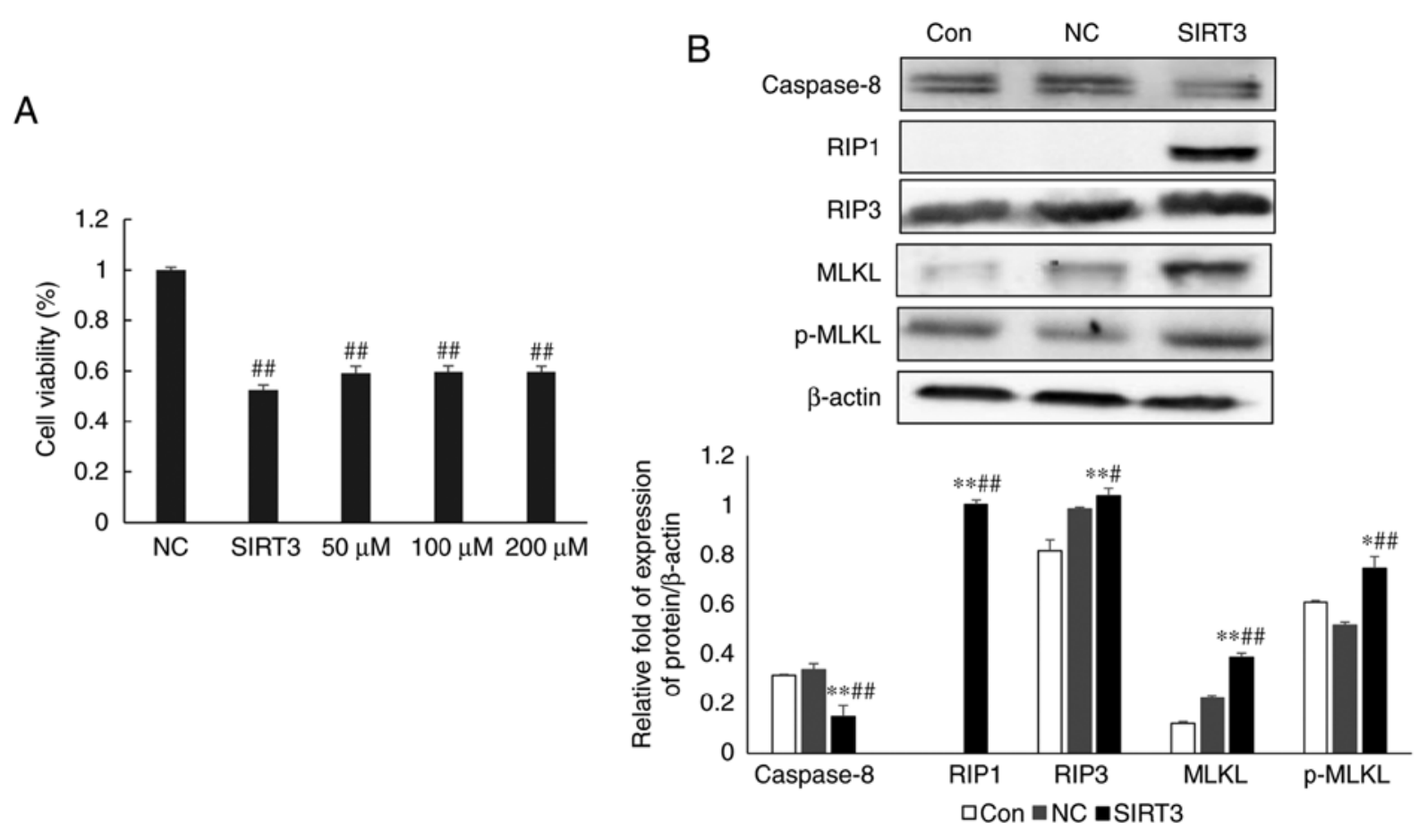

Figure 3. SIRT3 overexpression induces necrotic apoptosis in NCI-H446 small-cell lung cancer cells. (A) Effect of Nec-1 treatment on NCI-H446 cell survival, as determined by MTT assay. (B) Western blot analysis of caspase-8, RIP1, RIP3, MLKL and p-MLKL protein expression. "P<0.05 vs. the Con group; ${ }^{* *} \mathrm{P}<0.01$ vs. the Con group; ${ }^{\# P}<0.05$ vs. the NC group; ${ }^{* \#} \mathrm{P}<0.01$ vs. the NC group. SIRT3, Sirtuin 3 ; Nec-1, necrostatin-1.

SIRT3 inhibits NCI-H446 tumor growth in vivo. To further study the effects of SIRT3 on NCI-H446 cells, an in vivo experiment was conducted. NCI-H446 cells were injected subcutaneously into mice, and tumors were then allowed to establish and grow until approximately $5 \mathrm{~mm}$ in diameter. After 25 days, mice and tumors were photographed and differences in tumor size were evaluated. SIRT3 overexpression was revealed to inhibit tumor growth in vivo (Fig. $5 \mathrm{~A}$ and $\mathrm{B}$ ). The tumor growth index also confirmed that SIRT3 inhibited tumor growth (Fig. 5C). H\&E staining revealed increased apoptosis in the tumor tissues of the SIRT3 group (Fig. 5D). Immunohistochemical analysis demonstrated that SIRT3 protein expression was enhanced and that mutant p53 expression was decreased in the SIRT3 group, when compared with the PQ control group (Fig. 5E). Western blot analysis of tumor tissue extracts confirmed that SIRT3 treatment resulted in reduced mutant p53 expression (Fig. 5F), a finding that was consistent with our in vitro data, and which indicated that SIRT3 inhibited the development of small-cell lung cancer. Analysis of the expression of apoptosis-related proteins by western blotting revealed that the ratio of Bcl-2 to Bax protein in SIRT3 tumors was reduced, when compared with PQ control tumors (Fig. 5G). Cleaved caspase-3 expression was also upregulated in SIRT3 tumors. In addition, RT-PCR was used to evaluate changes in the expression of apoptosis-related genes (Fig. 5H). In agreement with the protein expression data, a decrease in $B C L 2$ to $B A X$ gene expression, and increased $C A S P 3$ gene expression was observed in SIRT3 tumors, when compared with controls.

\section{Discussion}

Researching the role of SIRT3 in tumors is becoming increasingly popular. However, Ashraf et al reported that increased
SIRT3 transcription was associated with node-positive breast cancer (14). The function of SIRT3 may therefore differ according to cell and tumor type. Consequently, SIRT3 may function both as an oncogene and a tumor suppressor gene.

In a study using A549 lung adenocarcinoma cells, SIRT3 was revealed to exhibit tumor suppressive properties in the regulation of cell proliferation. High SIRT3 expression was revealed to induce apoptosis through a mechanism involving increased Bax and Bad-dependent sequestration of Bc1-2 and Bcl-XL, respectively, thereby promoting AIF translocation to the nucleus. High expression of SIRT3 in A549 cells also resulted in an increase in p53 and p21 protein levels, and a decrease in the intracellular ROS level (15). We previously revealed that SIRT3 may also function as a tumor suppressor in small-cell lung cancer, a complex and highly malignant disease that lacks effective treatment options. It is therefore particularly important to explore the mechanism of the tumor suppressive function of SIRT3 in small-cell lung cancer, since this may facilitate the development of new therapeutic strategies for the treatment of this disease.

In the present study, it was demonstrated that SIRT3 can inhibit growth and promote apoptosis and necroptosis in NCI-H446 small-cell lung cancer cells using MTT and flow cytometric assays. In apoptosis mediated by the mitochondrial pathway, a change in the permeability of the outer mitochondrial membrane causes the release of cytochrome $c$. Cytoplasmic cytochrome $c$ activates Apaf-1, which further combines with procaspase-9 to form an apoptosis complex that activates procaspase- 9 . Once activated, caspase- 9 can activate other caspases, such as caspase-3. Mitochondrial membrane permeabilization is controlled by the $\mathrm{Bcl}-2$ protein family. One such member is Bax, an apoptosis-stimulating factor which is activated at the outer mitochondrial membrane to promote 
A
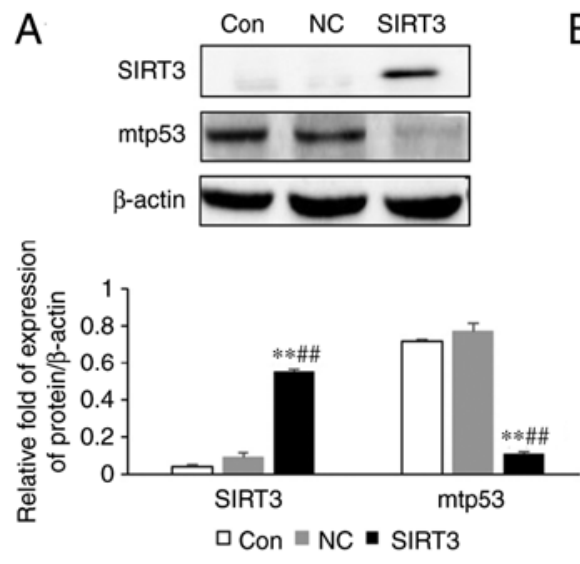

D
B
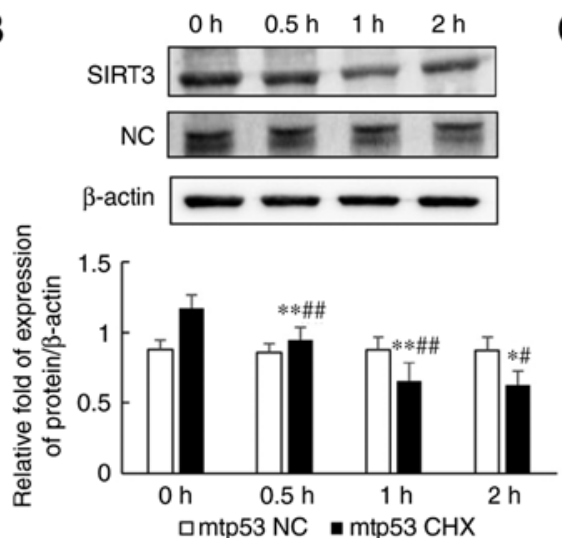

SIRT3+

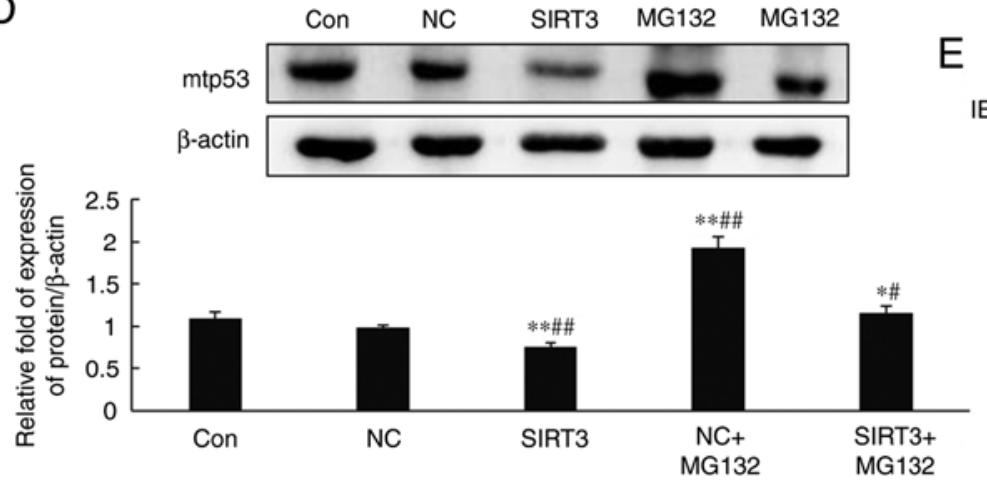

C

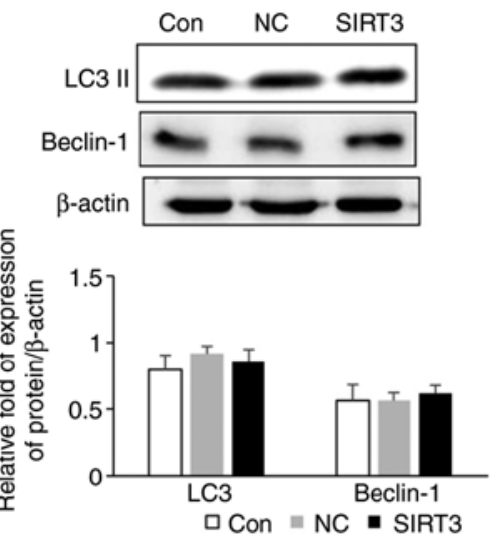

Figure 4. SIRT3-overepxression promotes degradation of mutant p53. (A) Western blot analysis of SIRT3 and mutant p53 protein levels in NCI-H446 small-cell lung cancer cells. (B) Comparison of the half-life of the mutant p53 protein with control after addition of CHX. (C) Protein expression levels of LC3 II and Beclin-1. (D) Western blot analysis of mutant p53 protein expression levels in NCI-H446 cells treated with or without MG132. (E) Analysis of mutant p53 protein ubiquitination by immunoprecipitation assay. ${ }^{*} \mathrm{P}<0.05$ vs. the Con group; ${ }^{* *} \mathrm{P}<0.01$ vs. the Con group; ${ }^{\#} \mathrm{P}<0.05$ vs. the $\mathrm{NC}$ group; ${ }^{\# \#} \mathrm{P}<0.01$ vs. the $\mathrm{NC}$ group. SIRT3, Sirtuin 3; CHX, cycloheximide.

membrane channel formation and release of cytochrome $c$. Bcl-2 can inhibit the activation of caspase- 9 by inhibiting the release of cytochrome $c$, and therefore functions as an inhibitor of apoptosis. In addition, MCL1 can inhibit Bax and thus antagonize apoptosis signaling, while Bid can promote mitochondrial membrane permeability, and thus is an activator of apoptosis. The present flow cytometric data revealed that SIRT3 overexpression induced cell apoptosis and necroptosis. Necroptosis is now considered as a regulated cellular process, an additional method of programmed cell death. The inhibition of caspase- 8 can activate receptor interaction protein kinase 1 (RIP1) and 3 (RIP3), and mixed lineage kinase domain-like (MLKL) which is regulated by RIP3 to be phosphorylated (p-MLKL). The activated MLKL is transferred from the cytoplasm to the cell membrane and the integrity of the cell membrane is damaged, resulting in cell necroptosis. $(16,17)$. In order to determine whether necroptosis also contributed to cellular death after SIRT3 overexpression, cells were treated with the necroptosis inhibitor, Nec-1, and cell survival was evaluated by MTT assay. MTT assays revealed that cell survival was increased in the presence of Nec-1, while western blotting assays revealed downregulation of caspase- 8 and upregulation of RIP1, RIP3, MLKL and p-MLKL. This inhibition of caspase- 8 and induction of RIP1, RIP3, MLKL and p-MLKL protein expression indicated that necrotic apoptosis was triggered after SIRT3 expression. In an in vivo model of small-cell lung cancer it was also revealed that SIRT3 expression could inhibit tumor growth through induction of tumor cell apoptosis. The present in vitro data indicated that a reduction in the ratio of $\mathrm{Bcl}-2$ to $\mathrm{Bax}$ and an associated activation of cleaved caspase- 3 provided the mechanism driving apoptosis in SIRT3-overexpressing cells.

Subsequently, the expression of mutant p53 after overexpression of SIRT3 in NCI-H446 cells was detected.

The results revealed that overexpression of SIRT3 could reduce the expression of mutant $\mathrm{p} 53$. In addition, it has been reported that the decrease of the level of mutant p53 can lead to an increase of apoptosis (18-20). The present study revealed for the first time that SIRT3 may promote cell apoptosis by leading to decreased levels of mutant $\mathrm{p} 53$. Studies indicated that SIRT3 can modify a large number of cellular proteins through it deacetylase activity. There are studies that have reported that SIRT3 can use its function of acetylation to modify many cellular proteins. Xiong et al revealed that, following SIRT3 overexpression in A549 cells, SIRT3 interacted with p53 and that these proteins co-localized in cells. Furthermore, SIRT3 was revealed to regulate the p53 degradation pathway by deacetylation (21). However, the mechanism by which SIRT3 regulates mutant $\mathrm{p} 53$ remains unknown.

TRRAP is a large multidomain protein that is a component of many histone acetyltransferase (HAT) multiprotein complexes. Silencing of TRRAP expression has been revealed to reduce p53 accumulation in lymphoma and colon cancer models. Conversely, overexpression of TRRAP has been 

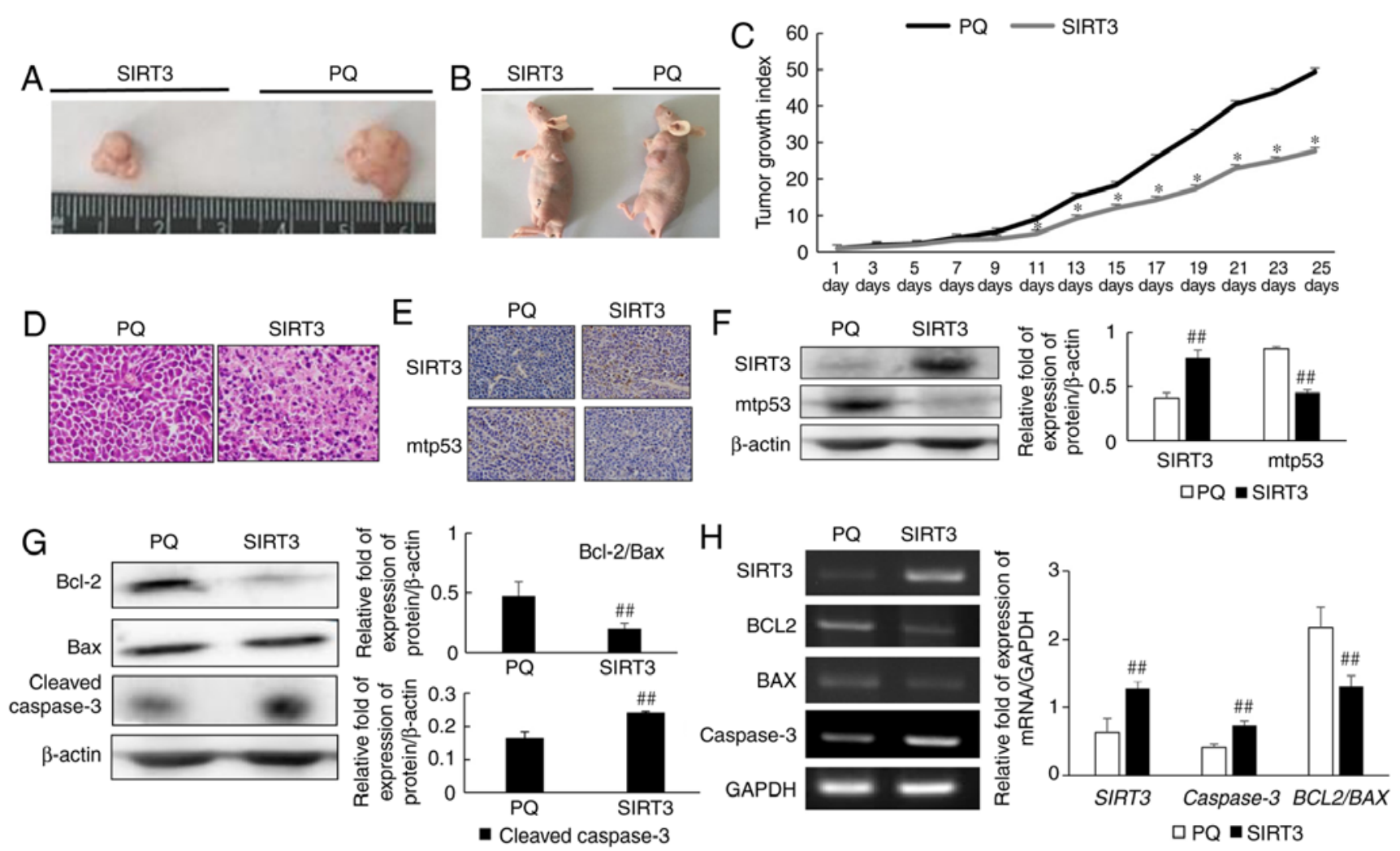

Figure 5. SIRT3 inhibits mutant p53 expression and impairs NCI-H446 tumor growth in vivo through activation of the apoptosis pathway. (A and B) Images of tumors and nude mice after sacrifice at day 25. (C) Tumor growth index values for each given treatment group recorded from the time of first bacterial inoculation. (D) H\&E staining of NCI-H446 xenograft tissue sections from animals inoculated with bacteria harboring control or SIRT3 plasmid (x20, magnification). (E) Immunohistochemical analysis of SIRT3 and mutant p53 expression in tumors harvested from animals inoculated with bacteria harboring control or SIRT3 plasmid. (F) Western blot analysis of SIRT3 and mutant p53 protein expression in tumors from animals inoculated with bacteria harboring control or SIRT3 plasmid. (G) Western blot analysis of cleaved caspase-3, Bcl-2 and Bax expression in tumors harvested from animals inoculated with bacteria harboring control or SIRT3 plasmid. (H) SIRT3, BCL2, BAX and CASP3 gene expression in tumors harvested from animals inoculated with bacteria harboring control or SIRT3 plasmid. The data are presented as the mean \pm standard deviation of triplicate experiments. ${ }^{*} \mathrm{P}<0.05$ vs. the $\mathrm{PQ}$ control; ${ }^{\# \#} \mathrm{P}<0.05$ vs. the $\mathrm{NC}$ group.

revealed to increase mutant p53 levels, while the inhibition of histone deacetylase (HDAC) has been revealed to decrease p53 levels (22). The present study therefore provides a link between deacetylation and mutant p53 stability.

SIRT3-mediated FOXO3 deacetylation has also been revealed to reduce FOXO3 phosphorylation, ubiquitination and degradation, thereby increasing protein stability (23). This suggests that SIRT3 activity may also influence the ubiquitination status of proteins.

Given these previous studies, it was hypothesized that the downregulation of mutant p53 observed in SIRT3overexpressing NCI-H446 cells was a consequence of the SIRT3-dependent deacetylation of mutant $\mathrm{p} 53$, which may impact the mutant p53 ubiquitination pathway. In NCI-H446 cells, both the expression and half-life of the mutant p53 protein were reduced following overexpression of SIRT3, a finding that indicated increased degradation of the protein. Protein degradation in eukaryotes is primarily mediated by the lysosomal and ubiquitin proteasome pathways. LC3 was the first protein to be identified as a marker of autophagy. Autophagosome formation requires the conversion of LC3 I into LC3 II (24) and, consequently, LC3 II levels increase during autophagy. Another autophagy-related protein, Beclin-1, which is the mammalian homolog of yeast Apg6/Vps30, forms a complex with the Class III PI3K Vps34, and together these proteins play an important role in activating autophagy (25). In the present study, it was revealed that LC3 II and Beclin-1 expression levels remained unchanged in SIRT3-overexpressing cells, indicating that the autophagy pathway was unlikely to be involved in the degradation of mutant p53. However, treatment of cells with the proteasome inhibitor MG132 resulted in a recovery of mutant p53 levels, while immunoprecipitation assays revealed that mutant p53 was ubiquitinated. Collectively, these results demonstrated that mutant p53 degradation was mediated by the ubiquitin proteasome pathway.

The present study revealed an inhibitory role for SIRT3 in small-cell lung cancer, whereby SIRT3 promoted cell apoptosis and necroptosis via a mechanism that may require degradation of mutant $\mathrm{p} 53$ by the ubiquitin proteasome degradation pathway. It is therefore possible that SIRT3-dependent loss of mutant p53 oncogene function provides a mechanism for inhibiting the development of small-cell lung cancer.

\section{Acknowledgements}

We would like to thank professor Xu Deqi of the Food and Drug Administration (FDA) for the donation of attenuated Salmonella typhimurium $\mathrm{phoP} / \mathrm{phoQ}$. We would also like to thank Dr James Monypenny, for editing the English text of a draft of this manuscript. 


\section{Funding}

The present study was supported by the Research Fund of the National Natural Science Foundation of China (grant nos. 81501982, 81572927, 81772794, 81472419 and 81672948), the Jilin Province Research Foundation for the Development of Science and Technology Project (grant nos. 20150414025GH, 20170623021TC and 20160414005GH) and the Jilin University Bethune Plan B Projects (grant no. 2015222), the Education Department of Jilin Province (no. JJKH20170834KJ), the Department of Science and Technology of Jilin Province, China (grant no. 20160520151JH) and the Department of Education of Jilin Province, China (grant no. 2016456).

\section{Availability of data and materials}

The datasets used during the present study are available from the corresponding author upon reasonable request.

\section{Authors' contributions}

XT, YLi, LS and YLiu participated in the research design. $\mathrm{XT}, \mathrm{LL}, \mathrm{RG}, \mathrm{PZ}$ and YiZ conducted the experiments and contributed to the data acquisition and analysis. YoZ, JZ and JS performed the data analysis. XT and YLi contributed to the writing of the manuscript. All authors read and approved the final manuscript.

\section{Ethics approval and consent to participate}

The animal study was approved by the Animal Ethics Review Committee of Basic Medical Sciences, Jilin University, in accordance with the regulations of the Institutional Committee for the Care and Use of Laboratory Animals of the Experimental Animal Center of Jilin University.

\section{Patient consent for publication}

Not applicable.

\section{Competing interests}

The authors declare that they have no competing interests.

\section{References}

1. Aridgides PD, Movsas B and Bogart JA: Thoracic radiotherapy for limited stage small cell lung carcinoma. Curr Probl Cancer 36 : 88-105, 2012

2. William WN Jr and Glisson BS: Novel strategies for the treatment of small-cell lung carcinoma. Nat Rev Clin Oncol 8: 611-619, 2011.

3. Nickolich M, Babakoohi S, Fu P and Dowlati A: Clinical trial design in small cell lung cancer: Surrogate end points and statistical evolution. Clin Lung Cancer 15: 207-212, 2014.

4. Byers LA and Rudin CM: Small cell lung cancer: Where do we go from here? Cancer 121: 664-672, 2015.
5. Xiong S, Tu H, Kollareddy M, Pant V, Li Q, Zhang Y, Jackson JG, Suh YA, Elizondo-Fraire AC, Yang P, et al: Pla2g16 phospholipase mediates gain-of-function activities of mutant p53. Proc Natl Acad Sci USA 111: 11145-11150, 2014

6. Nguyen GT, Schaefer S, Gertz M, Weyand M and Steegborn C: Structures of human sirtuin 3 complexes with ADP-ribose and with carba-NAD ${ }^{+}$and SRT1720: Binding details and inhibition mechanism. Acta Crystallogr D Biol Crystallogr 69: 1423-1432, 2013.

7. Guarente L: Introduction: Sirtuins in aging and diseases. Methods Mol Biol 1077: 3-10, 2013.

8. Zhang YY and Zhou LM: Sirt3 inhibits hepatocellular carcinoma cell growth through reducing Mdm2-mediated p53 degradation. Biochem Biophys Res Commun 423: 26-31, 2012.

9. Minamoto T, Buschmann T, Habelhah H, Matusevich E, Tahara H, Boerresen-Dale AL, Harris C, Sidransky D and Ronai Z: Distinct pattern of p53 phosphorylation in human tumors. Oncogene 20: 3341-3347, 2001.

10. Warnock LJ, Raines SA and Milner J: Aurora A mediates cross-talk between $\mathrm{N}$ - and C-terminal post-translational modifications of p53. Cancer Biol Ther 12: 1059-1068, 2011.

11. Rodriguez OC, Choudhury S, Kolukula V, Vietsch EE, Catania J, Preet A, Reynoso K, Bargonetti J, Wellstein A, Albanese C and Avantaggiati M: Dietary downregulation of mutant p53 levels via glucose restriction: Mechanisms and implications for tumor therapy. Cell Cycle 11: 4436-4446, 2012.

12. Pawelek JM, Low KB and Bermudes D: Bacteria as tumourtargeting vectors. Lancet Oncol 4: 548-556, 2003.

13. O'Leary EM and Igdoura SA: The therapeutic potential of pharmacological chaperones and proteosomal inhibitors, Celastrol and MG132 in the treatment of sialidosis. Mol Genet Metab 107: 173-185, 2012.

14. Ashraf N, Zino S, Macintyre A, Kingsmore D, Payne AP, George WD and Shiels PG: Altered sirtuin expression is associated with node-positive breast cancer. Br J Cancer 95: 1056-1061, 2006.

15. Xiao K, Jiang J, Wang W, Cao S, Zhu L, Zeng H, Ouyang R, Zhou R and Chen P: Sirt3 is a tumor suppressor in lung adenocarcinoma cells. Oncol Rep 30: 1323-1328, 2013.

16. Oberst A: Death in the fast lane: What's next for necroptosis? FEBS J 283: 2616-2625, 2016.

17. Pasparakis $M$ and Vandenabeele P: Necroptosis and its role in inflammation. Nature 517: 311-320, 2015.

18. Braicu C,Pileczki V, Irimie A and Berindan-Neagoe I: p53siRNA therapy reduces cell proliferation, migration and induces apoptosis in triple negative breast cancer cells. Mol Cell Biochem 381: 61-68, 2013.

19. Lim LY, Vidnovic N, Ellisen LW and Leong CO: Mutant p53 mediates survival of breast cancer cells. Br J Cancer 101: 1606-1612, 2009

20. Ali A, Wang Z, Fu J, Ji L, Liu J, Li L, Wang H, Chen J, Caulin C, Myers JN, et al: Differential regulation of the REGY-proteasome pathway by p53/TGF- $\beta$ signalling and mutant p53 in cancer cells. Nat Commun 4: 2667, 2013

21. Xiong Y, Wang L, Wang S, Wang M, Zhao J, Zhang Z, Li X, Jia L and Han Y: SIRT3 deacetylates and promotes degradation of P53 in PTEN-defective non-small cell lung cancer. J Cancer Res Clin Oncol 144: 189-198, 2018.

22. Jethwa A, Słabicki M, Hüllein J, Jentzsch M, Dalal V, Rabe S, Wagner L, Walther T, Klapper W; MMML Network Project, et al: TRRAP is essential for regulating the accumulation of mutant and wild-type p53 in lymphoma. Blood 131: 2789-2802, 2018.

23. Tseng AH, Wu LH, Shieh SS and Wang DL: SIRT3 interactions with FOXO3 acetylation, phosphorylation and ubiquitinylation mediate endothelial cell responses to hypoxia. Biochem J 464: 157-168, 2014.

24. Hemelaar J, Lelyveld VS, Kessler BM and Ploegh HL: A single protease, Apg4B, is specific for the autophagy-related ubiquitin-like proteins GATE-16, MAP1-LC3, GABARAP, and Apg8L. J Biol Chem 278: 51841-51850, 2003.

25. Boutouja F, Brinkmeier R, Mastalski T, El Magraoui F and Platta HW: Regulation of the tumor-suppressor BECLIN 1 by distinct ubiquitination cascades. Int J Mol Sci 18: E2541, 2017. 